\title{
Antimicrobial susceptibility pattern of Staphylococcus aureus isolates from clinical specimens at Kenyatta National Hospital
}

\author{
Wilfred Gitau ${ }^{1 *}$, Moses Masika', Moses Musyoki ${ }^{1}$, Beatrice Museve ${ }^{2}$ and Titus Mutwiri ${ }^{3}$
}

\begin{abstract}
Objective: To determine antibiotic susceptibility pattern of $S$. aureus isolates from clinical specimens collected from patients at Kenyatta National Hospital from March 2014-February 2016, and to determine the prevalence and quarterly trends of MRSA throughout the study period.

Results: A total of 944 S. aureus isolates were analyzed. High sensitivity of S. aureus was observed for quinupristin/ dalfopristin (100\%), tigecycline (98.2), imipenem (98\%), nitrofurantoin (97.6\%), linezolid (97.3\%), teicoplanin (97.1\%) and vancomycin (95.1\%). High resistance was recorded against penicillin G (91.9\%), trimethoprim/sulfamethoxazole (56.9\%) and tetracycline (33.2\%). MRSA prevalence among the patients at KNH was 27.8\%. Highest proportion (80\%) of MRSA was in burns unit. Both MRSA and MSSA were highly susceptible to quinupristin/dalfopristin, tigecycline, linezolid, nitrofurantoin, ampicillin/sulbactam and vancomycin and showed high resistance to commonly used antibiotics such as gentamycin, erythromycin, levofloxacin and tetracycline. A majority of isolates were from pus specimen (68\%).
\end{abstract}

Keywords: Methicillin resistant Staphylococcus aureus, Drug resistance, Susceptibility, Staphylococcus aureus, Vancomycin, Kenyatta National Hospital

\section{Introduction}

Staphylococcus aureus is a Gram-positive bacterium living as a commensal on the skin, mouth and upper respiratory system, making it a risk factor for opportunistic and nosocomial infections. Resistance to commonly used antimicrobial drugs is frequently encountered with $S$. aureus. Some of the mechanisms in resistance include; inactivation of antibiotics by the enzymes, decreased affinity for the antibiotics caused by alteration of the target, efflux pumps, and trapping of the antibiotic [1].

Staphylococcus aureus causes skin, bone, soft tissue infections, urinary tract infections, pneumonia, healthcare-associated bacteremia in community and hospital settings and other invasive infections. Multidrug-resistant strains particularly Multidrug Resistant Staphylococcus aureus (MRSA) strains are common causes of

\footnotetext{
*Correspondence: gwilfredrz@gmail.com

1 Department of Medical Microbiology, University of Nairobi, P.O Box 19676-00202, Nairobi, Kenya

Full list of author information is available at the end of the article
}

nosocomial infections and are associated with increased morbidity and mortality $[2,3]$.

Report from the National Nosocomial Infections Surveillance System of the Centers for Disease Control and Prevention, (2013-2015) showed that MRSA in India and USA accounts for $>60 \%$ of S. aureus isolates causing nosocomial infection in ICUs [4].

There is no conclusive local data on the magnitude of multidrug resistance MRSA infection burden in Kenyan hospitals. In study done from patients in select hospitals in Nairobi, MRSA infections were mostly isolated at public healthcare facilities serving economically disadvantaged Nairobi's population, like those living in urban informal settlements [5].

Another study done in Kenya showed there was high number of genetically indistinguishable isolates, which suggested there was local transmission of MSSA and MRSA [6].

Multidrug-resistant strains limit the therapeutic options, creating an economic and social burden to the healthcare system. Horizontal gene transfer in the 
hospital setting is responsible for disseminating antibiotic resistant determinants. Chromosomal mutation antibiotics selection is also responsible for antibiotics resistance.

The aim of this study was to evaluate the antimicrobial susceptibility pattern of Staphylococcus aureus at KNH from April 2014 to February 2016. Previous studies done in Kenya were targeting specific locations in the hospital and were within short periods of time. This study gives a broader picture of the susceptibility pattern of $S$ aureus in different locations in 3 years.

\section{Main text}

\section{Methodology}

This was a retrospective study based on electronic laboratory records of Staphylococcus aureus isolates from clinical specimens analyzed at KNH microbiology laboratory.

Data was retrieved from VITEK-2 Antibiotic Susceptibility Testing System, imported to WHONET software through BACLINK (World Health Organization). Analysis was done using WHONET and IBM SPSS V.21. VITEK 2 Gram Positive identification card (bioMerieux) was used to identify $S$. aureus sub-species aureus. All the isolates had an ID confidence of excellent identification with an average percent probability of $96 \%$.

Methicillin resistance was determined using cefoxitin screening.

All isolates were from patients' clinical specimens (mainly pus, urine, blood and tracheal aspirates) and were analyzed according to the 2015 Clinical \& Laboratory Standards Institute (CLSI M100-S25) standards.

Antibiotics tested against $S$. aureus include penicillin G (10 units), oxacillin (30 $\mu$ g cefoxitin), gentamycin $(10 \mu \mathrm{g})$, fusidic acid $(10 \mu \mathrm{g})$, cefuroxime $(30 \mu \mathrm{g})$, cefuroxime axetil $(30 \mu \mathrm{g})$, imipenem $(10 \mu \mathrm{g})$, tobramycin $(10 \mu \mathrm{g})$, rifampicin $(5 \mu \mathrm{g})$, levofloxacin $(5 \mu \mathrm{g})$, clindamycin $(2 \mu \mathrm{g})$, trimethoprim/sulfamethoxazole $(1.25 / 23.75 \mu \mathrm{g})$, moxifloxacin $(5 \mu \mathrm{g})$, nitrofurantoin $(300 \mu \mathrm{g})$, linezolid $(30 \mu \mathrm{g})$, vancomycin $(30 \mu \mathrm{g})$, teicoplanin $(30 \mu \mathrm{g})$, quinupristin/ dalfopristin $(15 \mu \mathrm{g})$, tetracycline $(30 \mu \mathrm{g})$, tigecycline $(15 \mu \mathrm{g})$, erythromycin $(15 \mu \mathrm{g})$ and ampicillin/sulbactam $(10 / 10 \mu \mathrm{g})$.

The sample size was calculated using Fishers formula [7]

$$
\begin{aligned}
\mathrm{n} & =\mathrm{z}^{2} \mathrm{pq}(1-\mathrm{q}) / \mathrm{d}^{2} \\
\mathrm{~N} & =1.96^{2} \times 0.47(1-0.47) / 0.05^{2}=382 .
\end{aligned}
$$

Univariate analysis was done using frequency distributions and proportions for categorical variance such as antimicrobial resistance and gender.

Bivariate analysis was done by use of Chi square to assess for association between categorical variables such as susceptibility of antibiotics and infections location. Data was presented in tables and graphs.

\section{Results}

A total of 944 S. aureus isolates were analyzed, 33\% (311/944) of pathogens were isolated in 2014, 62\% (586/944) in 2015 and 5\% (47/944) in 2016. Majority of the analyzed isolates, $54 \%(511 / 944)$, were from male patients.

Internal Medicine department recorded the highest number of isolates, $187 / 944$ (20\%). A majority of the isolates were from pus specimen, 638/944 (68\%), tracheal aspirate $(15 \%)$ and blood (11\%). Other specimen types, 26/944 (3\%) included tissue, sputum, eye, throat, CSF and the unindicated specimens.

High susceptibility was seen with quinupristin/dalfopristin (100\%). High resistance was observed with penicillin G (92\%) and trimethoprim/sulfamethoxazole (57\%) (Table 1).

Isolates from pus to HVS specimens showed high susceptibility to ampicillin-sulbactam (100\%) while isolates from blood showed least susceptibility (90\%) Isolates

\begin{tabular}{|c|c|c|c|}
\hline Antibiotic & No. of isolates & $\% S$ & $\%$ NS \\
\hline Quinupristin/dalfopristin & 98 & 100 & 0 \\
\hline Ampicillin/sulbactam & 98 & 98 & 2 \\
\hline Imipenem & 98 & 98 & 2 \\
\hline Nitrofurantoin & 929 & 98 & 2 \\
\hline Tigecycline & 925 & 98 & 2 \\
\hline Linezolid & 929 & 97 & 3 \\
\hline Teicoplanin & 929 & 97 & 3 \\
\hline Fosfomycin & 791 & 96 & 4 \\
\hline Vancomycin & 927 & 95 & 5 \\
\hline Clindamycin & 928 & 93 & 7 \\
\hline Rifampin & 830 & 92 & 8 \\
\hline Fusidic acid & 791 & 88 & 12 \\
\hline Gentamicin & 831 & 87 & 13 \\
\hline Tobramycin & 831 & 87 & 13 \\
\hline Cefuroxime & 98 & 85 & 15 \\
\hline Cefuroxime axetil & 98 & 85 & 15 \\
\hline Mupirocin & 831 & 82 & 18 \\
\hline Levofloxacin & 929 & 78 & 22 \\
\hline Moxifloxacin & 929 & 78 & 22 \\
\hline Erythromycin & 930 & 73 & 26 \\
\hline Cefoxitin screen & 831 & 72 & 28 \\
\hline Oxacillin & 828 & 71 & 29 \\
\hline Tetracycline & 929 & 67 & 33 \\
\hline Trimethoprim/sulfamethoxazole & 929 & 43 & 57 \\
\hline Penicillin G & 829 & 8 & 92 \\
\hline
\end{tabular}

Table 1 Antimicrobial susceptibility profile

S Susceptible; NS non susceptible (resistant and intermediate) 
from pus specimen recorded high resistance to cephalosporins (17\%). Isolates from all specimens showed high susceptibility to imipenem.

Staphylococcus aureus isolates from HVS showed high resistance to tobramycin (16\%) and gentamycin (24\%), but showed high susceptibility to quinupristin/dalfopristin (100\%), cefuroxime (100\%) and imipenem (100\%) High susceptibility to quinolones was observed in isolates from other specimens (90\%) and low susceptibility was observed in isolates from tracheal aspirates (75\%).

As shown in Table 1, isolates from all the specimens showed $100 \%$ sensitivity to quinupristin/dalfopristin. Isolates from HVS showed high resistance to tetracycline (41\%), erythromycin (34\%) and clindamycin (19\%), no resistance was recorded from isolates from CSF to clindamycin.

Nine per cent of isolates from tracheal aspirates recorded complete resistance to vancomycin (VRSA) and 3\% recorded intermediate resistance to vancomycin (VISA). Isolates from tracheal aspirates also showed lower susceptibility to teicoplanin (93\%). S. aureus isolates from CSF (100\%) and pus (99\%) recorded high susceptibility to tigecycline.

Isolates from pus specimens recorded high resistance to trimethoprim/sulfamethoxazole (60\%). High resistance to linezolid was observed in isolates from tracheal aspirates (7\%). Isolates from HVS recorded high resistance to fosfomycin (24\%), mupirocin (36\%) and nitrofurantoin (6\%).

\section{MRSA distribution by year}

The overall prevalence of MRSA was 27.8\%. MRSA prevalence for 2014 was $34.9 \%$ (103/298) and 2015 recorded 25.8\% (124/481). 2016 had MRSA prevalence of $21.7 \%$ representing samples for 2 months (January and February). There was no significant difference between MRSA isolation and the year of isolation $(\mathrm{P}=0.159)$.

\section{MRSA Distribution by specimen}

The highest prevalence of MRSA was observed in pus specimen 153/232 (66\%).

\section{Quarterly trend of MRSA}

A high proportion of MRSA, 48/127 (38\%), was observed in the fourth quarter (Q4) of 2014 as shown in Fig. 1. Least proportion, $18 / 110$ (16\%) was observed in the third quarter of 2015 (Fig. 1).

\section{Susceptibility of MRSA to different antibiotics}

High susceptibility of MRSA isolates was observed with tigecycline (97\%), nitrofurantoin (96\%), linezolid (95.3\%), teicoplanin (94.8\%) and vancomycin (94.7\%). This is shown in Table 2.

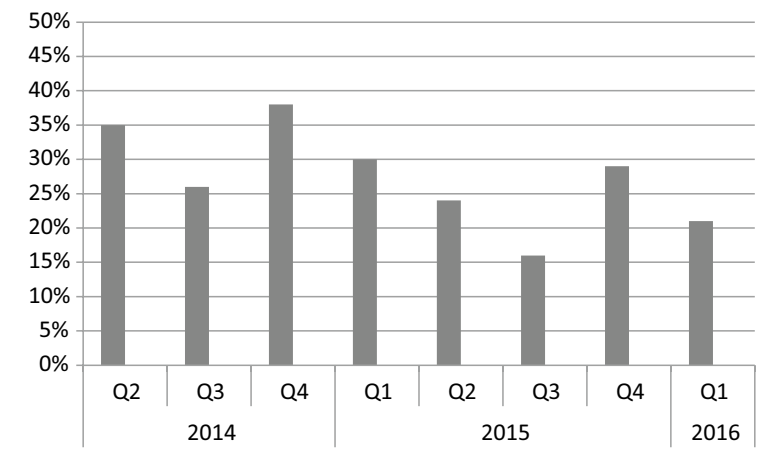

Fig. 1 Quarterly trend of MRSA (No. 944). Q Quarter

\section{Discussion}

The aim of this study was to evaluate the antimicrobial susceptibility pattern of $S$. aureus from clinical specimens at Kenyatta National Hospital. In our study, most S. aureus strains (68\%) were isolated from pus specimen. This is consistent with a previous study done at KNH [8]. In a study done to determine the antimicrobial susceptibility pattern of $S$. aureus strains isolated from hospitalized patients in Iran, most of the isolates were from blood specimens (29\%) [9]. Another study done on prevalence and antibiotic susceptibility pattern of $S$. aureus from clinical isolates in Nigeria showed a majority of the isolates were from urine specimens (76\%) [10].

The high number of $S$. aureus isolated in pus may be attributed to exposure of wounds which makes them more prone to infections and poor hygiene.

Staphylococcus aureus isolates showed high sensitivity to quinupristin/dalfopristin, imipenem, nitrofurantoin, tigecycline, ampicillin/sulbactam and linezolid. This is consistent with a similar study done in Iran [11]. Research done on antibiotics currently used in the treatment of infections caused by S. aureus in Australia indicates quinupristin/dalfopristin and linezolid have good antistaphylococcal activity but are very expensive [12].

In our study, high resistance of $S$. aureus isolates was observed against penicillin G (91.9\%), and trimethoprim/ sulfamethoxazole (56.9\%). This finding is similar to those of studies done at KNH [13], in Eritrea [14], in Nigeria [15], and Namibia [16]. This resistance could be attributed to the mechanism of resistance like the permeability barrier, efflux pumps, mutational or recombinational changes in the target enzymes and acquired resistance by drug-resistant target enzymes in trimethoprim/sulfamethoxazole and alteration of the target with decreased affinity for the antibiotic in penicillin [1].

In this study, MRSA was tested using cefoxitin screening. Overall MRSA prevalence was $27.8 \%$. This prevalence was lower than in previous studies that reported 
Table 2 Susceptibility of MRSA to different antibiotics

\begin{tabular}{|c|c|c|}
\hline Antibiotic & No. of MRSA & $\%$ Susceptible \\
\hline Tigecycline & 230 & 97 \\
\hline Nitrofurantoin & 231 & 96 \\
\hline Linezolid & 232 & 95 \\
\hline Teicoplanin & 229 & 95 \\
\hline Vancomycin & 228 & 95 \\
\hline Fosfomycin & 220 & 92 \\
\hline Clindamycin & 221 & 86 \\
\hline Rifampicin & 230 & 81 \\
\hline Tobramycin & 219 & 73 \\
\hline Fusidic acid & 220 & 72 \\
\hline Gentamycin & 218 & 66 \\
\hline Moxifloxacin & 174 & 58 \\
\hline Mupirocin & 231 & 56 \\
\hline Levofloxacin & 228 & 44 \\
\hline Erythromycin & 205 & 37 \\
\hline Tetracycline & 231 & 35 \\
\hline $\begin{array}{l}\text { Trimethoprim/sulfameth- } \\
\text { oxazole }\end{array}$ & 232 & 25 \\
\hline Oxacillin & 229 & 25 \\
\hline Penicillin G & 229 & 3 \\
\hline
\end{tabular}

46.5\% of MRSA prevalence at KNH [13], 31.5\% in Uganda [17] and 46.3\% in Iran [18].

This difference could be attributable to interventions that may have been effected during the study period such as infection control and improved antimicrobial stewardship and/or appropriate antibiotic use, this difference in MRSA prevalence could also be attributed to the different laboratory techniques used to correctly identify MRSA. KNH started using automated VITEK-2 system in mid 2013. VITEK ${ }^{@} 2$ Gram Positive identification card (vitek ${ }^{@} 2$ gp card bioMerieux) used at $\mathrm{KNH}$ is sensitive and specific to subspecies $S$. aureus aureus $[18,19]$.

In contrast, MRSA prevalence was lower in studies done in two private hospitals in Nairobi, Kenya, which showed a $3.8 \%$ prevalence [20]; a study in Eritrea that recorded $9 \%$ prevalence and $0.03 \%$ prevalence in Dutch hospitals $[13,21]$. The low prevalence of MRSA in private hospitals could be attributed to better infection controls. This shows there is high variance of MRSA prevalence from different countries. Majority of MRSA was isolated from pus specimens, 154/232 (66\%). Our finding concurs with studies done in two private hospitals in Nairobi, a Namibian institute of pathology and a tertiary health institution in Nigeria $[14,15,22]$. In contrast, other studies done in Nigeria, Iran and Jamaica showed different specimens were predominant $[9,23,24]$. The high number of MRSA from pus in our study could be due to exposure of wounds and abscesses to $S$. aureus. Carriage of $S$. aureus on the skin makes wounds more prone to MRSA infections.

In this study, $5 \%$ of MRSA isolates were resistant to vancomycin. This finding is similar to a study done in Iran which showed $5 \%$ of the MRSA isolates were resistant to vancomycin [11]. This contrasts similar studies done in a tertiary care hospital in India and pediatrics and neonatal intensive care patients at $\mathrm{KNH}$ which, respectively showed 3.5 and $1 \%$ resistance to vancomycin among MRSA [12, 23]. Studies done on antimicrobial susceptibility of MRSA in hospitalized patients in Iran, two hospitals in India and two private hospitals in Kenya showed $100 \%$ susceptibility to vancomycin $[8,22,25]$. Our study showed $S$. aureus isolates were highly susceptible to newer drugs. These drugs include; quinupristin/dalfopristin, tigecycline, imipenem, teicoplanin, vancomycin and linezolid. Similar studies done in Kenya and USA have shown $S$. aureus to be highly susceptible to ceftobiprole, tigecycline, linezolid, teicoplanin, vancomycin and daptomycin $[22,26]$.

This finding differs from a study done by Arianpoor et al. on antimicrobial susceptibility pattern of $S$. aureus isolates against newly marketed antibiotics in Iran which showed $5.5 \%$ of MRSA isolates were resistant to linezolid, $5.9 \%$ of to quinupristin-dalfopristin and $18.9 \%$ to tigecycline [11].

\section{Conclusion and recommendations}

Our study showed that burns unit had the highest (80\%) proportion of MRSA isolates. Both MRSA and MSSA were highly susceptible to quinupristin/dalfopristin, tigecycline, linezolid, nitrofurantoin, ampicillin/sulbactam, and vancomycin, but showed high resistance to commonly used antibiotics such as gentamycin, erythromycin, levofloxacin, SMX-TMP and tetracycline. Infection control measures should be enhanced in burns unit. Information from this study may be used in future as a baseline for follow-up to the susceptibility trend of various drugs to be used for the treatment of $S$. aureus infections. Routine screening of MRSA and regular studies should be conducted to find out the sources of MRSA. It is important to do culture and sensitivity of relevant specimens when $S$ aureus infection is suspected. There is need for further research on molecular studies evaluating the resistance genes and monitoring the epidemiology of multiple drug resistant $S$. aureus and MRSA.

\section{Limitations}

This was a retrospective study, where some information such as specimen type, collection date, age of the patient, clinical information, previous antibiotics use, duration of patient stay in the hospital and outcome of the therapy were missing. 


\section{Abbreviations}

Bla: beta-lactamase; CA-MRSA: community associated methicillin resistant Staphylococcus aureus; CSF: cerebrospinal fluid; HA-MRSA: hospital associated methicillin resistant Staphylococcus aureus; ICU: intensive care unit; KNH: Kenyatta National Hospital; MRSA: methicillin resistant Staphylococcus aureus; NICU: neonatal intensive care unit; PBP: penicillin binding proteins; PICU: pediatric intensive care unit; SOP: standard operating procedure; UoN: University of Nairobi; VISA: vancomycin intermediate Staphylococcus aureus; VRSA: vancomycin resistant Staphylococcus aureus; WHO: World Health Organization.

\section{Authors' contributions}

GW, MM1 and TM were involved in study conception and design. GW, MM, $\mathrm{TM}, \mathrm{MM} 2$ and $\mathrm{BM}$ were involved with drafting, reading. All authors read and approved the final manuscript.

\section{Author details}

1 Department of Medical Microbiology, University of Nairobi, P.O Box 19676-00202, Nairobi, Kenya. ${ }^{2}$ Department of Laboratory Medicine, Microbiology, Kenyatta National Hospital, P.O Box 20723-00202, Nairobi, Kenya. ${ }^{3}$ Department of Medical Laboratory, Kenya Methodist University, P.O Box 45240-00100, Nairobi, Kenya.

\section{Acknowledgements}

The authors would like to acknowledge; KNH's microbiology laboratory manager for allowing us to collect data from their archives and all the staff at the department of Medical microbiology, University of Nairobi, for their support during this study.

\section{Competing interests}

The authors declare that they have no competing interests.

\section{Availability of data and materials}

We are unable to make public the data available due to participants' confidentiality.

\section{Consent for publication}

Not applicable.

\section{Ethics approval and consent to participate}

Study approval was obtained from KNH/UoN Ethics and Research Committee (UP26/01/2016) and permission to collect the data was sought from the Assistant Director, Laboratory Medicine, $\mathrm{KNH}$.

A waiver of patient consent was sought since the research was based on secondary data from the laboratory.

\section{Funding}

The study was solely funded by authors.

\section{Publisher's Note}

Springer Nature remains neutral with regard to jurisdictional claims in published maps and institutional affiliations.

Received: 8 February 2018 Accepted: 28 March 2018

Published online: 03 April 2018

\section{References}

1. Pantosti A, Sanchini A, Monaco M. Mechanisms of antibiotic resistance in Staphylococcus aureus. Future Microbiol. 2007;2:323-34.

2. Loomba PS, Taneja J, Mishra B. Methicillin and vancomycin resistant S. aureus in hospitalized patients. J. Glob Infect Dis. 2010;2:275-83.

3. Espedido BA, Gosbell IB. Chromosomal mutations involved in antibiotic resistance in Staphylococcus aureus. Front Biosci. 2012;4:900-15.

4. Kumar M. Multidrug-resistant Staphylococcus aureus, India, 2013-2015. Emerg Infect Dis. 2016;22:1666-7.

5. Maina EK, Kiiyukia C, Wamae CN, Waiyaki PG, Kariuki S. Characterization of methicillin-resistant Staphylococcus aureus from skin and soft tissue infections in patients in Nairobi, Kenya. Int J Infect Dis. 2013;17:e115-9.
6. Aiken AM, Mutuku IM, Sabat AJ, Akkerboom V, Mwangi J, Scott JAG, et al. Carriage of Staphylococcus aureus in Thika Level 5 Hospital, Kenya: a crosssectional study. Antimicrob Resist Infect Control. 2014;3:22.

7. Israel GD. Determining sample size 1. Gainesville: Inst. Food Agric. Sci., Univ. Florida; 1992. p. 1-5.

8. Kanaga EL. Antimicrobial susceptibility of bacteria that cause wound sepsis in the paediatric surgical patients at Kenyatta national hospital. 2014. http://erepository.uonbi.ac.ke/handle/11295/95412. Accessed 7 Sept 2017.

9. Soltani R, Khalili H, Rasoolinejad M, Abdollahi A. Antimicrobial susceptibility pattern of Staphylococcus aureus strains isolated from hospitalized patients in Tehran, Iran. Iran J Pharm Sci. 2010;6:125-32.

10. Obiazi HAK, Ekundayo AO, Ukwandu NCD. Prevalence and antibiotic susceptibility pattern of Staphylococcus aureus from clinical isolates grown at 37 and $44^{\circ} \mathrm{C}$ from Irrua, Nigeria. African J Microbiol Res. 2007;1:57-60.

11. Arianpoor A, Estaji F, Naderinasab M, Askari E. Antimicrobial susceptibility pattern of Staphylococcus aureus Isolates against newly marketed antibiotics. J Ayub Med Coll Abbottabad. 2015:3:3-6.

12. Rayner C, Munckhof WJ. Antibiotics currently used in the treatment of infections caused by Staphylococcus aureus. Intern Med J. 2005;35(Suppl 2):3-16.

13. Rutare S. Prevalence of methicillin resistant Staphylococcus aureus (MRSA) among paediatric patients admitted in intensive care unit and neonatal intensive care unit at Kenyatta national hospital-Nairobi. Kenya: University of Nairobi; 2013.

14. Naik D, Teclu A. A study on antimicrobial susceptibility pattern in clinical isolates of Staphylococcus aureus in Eritrea. Pan Afr Med J. 2009:3:1.

15. Nwankwo EO, Nasiru MS. Antibiotic sensitivity pattern of Staphylococcus aureus from clinical isolates in a tertiary health institution in Kano, Northwestern Nigeria. Pan Afr Med J. 2011;8:4.

16. lileka AEK, Mukesi M, Engelbrecht F, Moyo SR. Antimicrobial susceptibility patterns of Staphylococcus aureus strains isolated at the Namibia Institute of Pathology from 2012 to 2014. Open J Med Microbiol. 2012;2016:116-24.

17. Ojulong J, Mwambu TP, Joloba M, Bwanga F, Kaddu-Mulindwa DH. Relative prevalence of methicilline resistant Staphylococcus aureus and its susceptibility pattern in Mulago Hospital, Kampala, Uganda. Tanzan J Health Res. 2009:11:149-53.

18. Dibah S, Arzanlou M, Jannati E, Shapouri R. Prevalence and antimicrobial resistance pattern of methicillin resistant Staphylococcus aureus (MRSA) strains isolated from clinical specimens in Ardabil, Iran. Iran J Microbiol. 2014;6:163-8.

19. Funke G, Funke-Kissling P. Performance of the new VITEK 2 GP card for identification of medically relevant Gram-positive cocci in a routine clinical laboratory. J Clin Microbiol. 2005;43:84-8.

20. Omuse G, Kabera B, Revathi G. Low prevalence of methicillin resistant Staphylococcus aureus as determined by an automated identification system in two private hospitals in Nairobi, Kenya: a cross sectional study. BMC Infect Dis. 2014:14:669.

21. Wertheim HFL, Vos MC, Boelens HAM, Voss A, Vandenbroucke-Grauls CMJE, Meester MHM, et al. Low prevalence of methicillin-resistant Staphylococcus aureus (MRSA) at hospital admission in the Netherlands: the value of search and destroy and restrictive antibiotic use. J Hosp Infect. 2004;56:321-5.

22. Crowley E, Bird P, Fisher K, Goetz K, Boyle M, Benzinger MJJ, et al. Evaluation of the VITEK 2 gram positive (GP) microbial identification test card: collaborative study. J AOAC Int. 2012;95:1425-32.

23. Mir BA. Prevalence and antimicrobial susceptibility of methicillin resistant Staphylococcus aureus and coagulase-negative Staphylococci in a tertiary care hospital. Asian J Pharm Clin Res. 2013;6:23-6.

24. Brown PD, Ngeno C. Antimicrobial resistance in clinical isolates of Staphylococcus aureus from hospital and community sources in southern Jamaica. Int J Infect Dis. 2007;11:220-5.

25. Joshi S, et al. Methicillin resistant Staphylococcus aureus (MRSA) in India: prevalence \& susceptibility pattern. Indian J Med Res. 2013;137:363-9.

26. Moreillon P. New and emerging treatment of Staphylococcus aureus infections in the hospital setting. Clin Microbiol Infect. 2008;14(Suppl 3):32-41. 